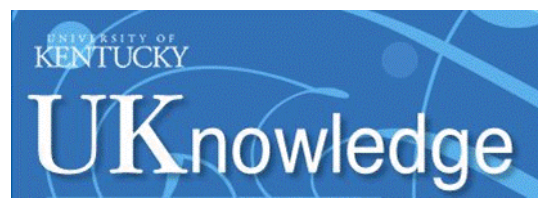

University of Kentucky

UKnowledge

$12-29-2021$

\title{
Numerical Investigation on the Effect of Spectral Radiative Heat Transfer within an Ablative Material
}

\author{
Raghava S. C. Davuluri \\ University of Kentucky, raghava.sai.chaitanya@gmail.com \\ Rui Fu \\ University of Kentucky, rick.fu@uky.edu \\ Kaveh A. Tagavi \\ University of Kentucky, kaveh.tagavi@uky.edu \\ Alexandre Martin \\ University of Kentucky, alexandre.martin@uky.edu
}

Follow this and additional works at: https://uknowledge.uky.edu/me_facpub

Part of the Aerodynamics and Fluid Mechanics Commons, Heat Transfer, Combustion Commons, Materials Science and Engineering Commons, Numerical Analysis and Computation Commons, Structures and Materials Commons, Thermodynamics Commons, and the Transport Phenomena Commons

Right click to open a feedback form in a new tab to let us know how this document benefits you.

\section{Repository Citation}

Davuluri, Raghava S. C.; Fu, Rui; Tagavi, Kaveh A.; and Martin, Alexandre, "Numerical Investigation on the Effect of Spectral Radiative Heat Transfer within an Ablative Material" (2021). Mechanical Engineering Faculty Publications. 80.

https://uknowledge.uky.edu/me_facpub/80

This Conference Proceeding is brought to you for free and open access by the Mechanical Engineering at UKnowledge. It has been accepted for inclusion in Mechanical Engineering Faculty Publications by an authorized administrator of UKnowledge. For more information, please contact UKnowledge@lsv.uky.edu. 
Numerical Investigation on the Effect of Spectral Radiative Heat Transfer within an Ablative Material

Digital Object Identifier (DOI)

https://doi.org/10.2514/6.2022-1283

Notes/Citation Information

Published in AIAA Scitech 2022 Forum.

Copyright $\odot 2022$ by Raghava S. C. Davuluri, Rui Fu, Kaveh A. Tagavi, Alexandre Martin.

The copyright holders have granted the permission for posting the article here.

This conference proceeding is available at UKnowledge: https://uknowledge.uky.edu/me_facpub/80 


\title{
Numerical investigation on the effect of spectral radiative heat transfer within an ablative material
}

\author{
Raghava S.C. Davuluri, Rui Fu† Kaveh A. Tagavi $\ddagger$ and Alexandre Martin§, \\ University of Kentucky, Lexington, KY, 40506
}

\begin{abstract}
The spectral radiative heat flux could impact the material response. In order to evaluate it, a coupling scheme between KATS - MR and $P_{1}$ approximation model of radiation transfer equation (RTE) is constructed and used. A Band model is developed that divides the spectral domain into small bands of unequal widths. Two verification studies are conducted: one by comparing the simulation computed by the Band model with pure conduction results and the other by comparing with similar models of RTE. The comparative results from the verification studies indicate that the Band model is computationally efficient and can be used to simulate the material's response when exposed to spectral radiative heat flux. To further evaluate the effectiveness of the spectral form of heat transfer, material response simulations were run by taking into account spectral data as the boundary condition. The results indicate a significant difference in temperature and density distributions within the sample. The internal temperatures are predicted higher with early decomposition when the spectral radiative heat flux is considered.
\end{abstract}

\section{Introduction}

High surface heat fluxes are experienced by space vehicles while entering a planetary atmosphere at hypersonic speeds. The convective heat flux is contributed by the high enthalpy of the flow, whereas the radiative heat flux is from shock layer radiation. The vehicle is thus equipped with Thermal Protection System (TPS) to counter the heat. Ablative fibrous materials are the most commonly used TPS materials that remove the heat by undergoing mass-removal mechanisms. ${ }^{1}$

The thermal response of the material to the high heat is in the form of heat conduction and internal radiation through the porous material. The material response models use effective thermal conductivity to model both forms of heat transfer within the material. ${ }^{2}$ The effect of radiation on the material is accounted for by taking the total radiative flux as a boundary condition. Therefore, the spectral form of radiative heat flux is assumed to be ineffective to material response and was not considered for modeling. However, recent experiments from White ${ }^{3}$ indicate otherwise.

White $^{3}$ had conducted laser irradiation experiments on carbon and silica fiber felts. Two types of lasers, $\mathrm{CO}_{2}$ laser and Fiber laser, were used to irradiate samples for 30 seconds that impose a heat flux of $115 \mathrm{~W} / \mathrm{cm}^{2}$ on the surface. The experimental observations indicated that the material response to the heating was different when the samples irradiated by the two lasers were compared. It was seen that char layer thickness, mass loss, bondline temperature, time to reach the maximum bondline temperature were higher for samples irradiated by Fiber laser when compared to $\mathrm{CO}_{2}$ laser. It was concluded that wavelength-dependent radiative effects could impact on the material response.

In the earlier work, ${ }^{4}$ the internal radiation and heat conduction were solved separately and coupled to account for the spectral contribution of the radiative heat transfer. A $\mathrm{P}_{1}$ approximation model was used to compute the internal radiation of the material. The coupling strategy was validated on multi-dimensional meshes, indicating an accurate material response prediction. The work was extended to simulate the laser ablation mimicking White ${ }^{3}$ experiments, which showed the expected behavior as reported by White. ${ }^{3}$

\footnotetext{
*Graduate Student, Mechanical Engineering, AIAA Student Member

${ }^{\dagger}$ Research Scientist, Mechanical Engineering, AIAA Member

${ }^{\ddagger}$ Professor, Mechanical Engineering

§Professor, Mechanical Engineering, Associate Fellow AIAA. alexandre.martin@uky.edu
} 
The shock layer radiation can be expressed in terms of narrow bands of emission, ${ }^{5}$ and therefore, the effect of spectral radiative heat flux on the material response should be investigated to model the TPS accurately. Therefore, the present work examines the material's response to spectral heat flux.

\section{Methodology}

\section{II.A. Numerical Models}

\section{II.A.1. KATS - MR}

A finite volume solver, KATS (Kentucky Aerothermodynamics and Thermal-response Solver), ${ }^{6-12}$ is used to simulate the response of the material. The governing equation of the solver is of the form:

$$
\frac{\partial \mathbf{Q}}{\partial t}+\nabla \cdot\left(\mathcal{F}-\mathcal{F}_{\boldsymbol{d}}\right)=\mathbf{S},
$$

where $\mathbf{Q}$ is a vector of conservative variables, $\mathcal{F}$ and $\mathcal{F}_{\boldsymbol{d}}$ are convective and diffusive flux matrices, and $\mathbf{S}$ is the source term vector. The discretization of Eq. 1 is performed first order in time and second order in space. The solver uses the PETSc library ${ }^{13-15}$ to solve the system of equations, ParMETIS ${ }^{16}$ for domain decomposition, and $\mathrm{MPI}^{17}$ for message passing. More information on this solver is presented in the works by Weng et al. ${ }^{6,18,19}$

\section{II.A.2. Radiation Transfer Equation}

A $\mathrm{P}_{1}$ approximation version of Radiative Transfer Equation (RTE) ${ }^{20}$ is used to solve the radiation energy balance within the material. The $\mathrm{P}_{1}$ approximation of RTE yields two relationships between spectral incident radiative flux $\left(G_{\lambda}\right)$ and spectral radiative heat flux $\left(q_{\lambda}\right)$ which are given as:

$$
\begin{gathered}
\nabla \cdot q_{\lambda}=\kappa_{\lambda}\left(4 \pi I_{b \lambda}-G_{\lambda}\right) \\
q_{\lambda}=-\frac{1}{3 \beta_{\lambda}} \nabla G_{\lambda}
\end{gathered}
$$

where $I_{b \lambda}, \kappa_{\lambda}, \beta_{\lambda}$ represents Planck function, absorption and extinction coefficients, respectively. A Helmholtz equation is formed when Eq. 2 and Eq. 3 are combined which is given as:

$$
\nabla \cdot\left(-\frac{1}{3 \beta_{\lambda}} \nabla G_{\lambda}\right)=\kappa_{\lambda}\left(4 \pi I_{b \lambda}-G_{\lambda}\right)
$$

\section{II.B. Solution procedure}

The general form for the energy part of Eq. 1 for a heat conduction problem is given as:

$$
\rho_{s} c_{p} \frac{\partial T}{\partial t}=\nabla \cdot k_{\mathrm{eff}} \nabla T
$$

where $\rho_{s}$ is the total solid density of the material, $c_{p}$ is specific heat capacity of solid, $T$ is the temperature, $t$ is time, and $k_{\text {eff }}$ is the effective thermal conductivity. The heat conduction through the solid material solved by using Eq. 5 is referred to as "pure conduction" in this work. The effective thermal conductivity takes into account the thermal conductivities of solid, gas, as well as radiation. The effect of shock layer radiation on the material is evaluated by solving Eq. 5 with a total radiative flux as a boundary condition. In order to investigate the effect of individual spectral radiative fluxes on the material, a coupled approach between heat conduction and RTE should be used. The modified version of the energy equation used for the coupling scheme is given as:

$$
\rho_{s} c_{p} \frac{\partial T}{\partial t}=\nabla \cdot\left(k_{s / g} \nabla T+q_{\mathrm{rad}}\right)=\nabla \cdot\left(k_{s / g} \nabla T\right)+\nabla \cdot q_{\mathrm{rad}}
$$


where $k_{s / g}$ is the thermal conductivity that takes into account the contributions of solid and gas phase, and $q_{\text {rad }}$ is the total radiative heat flux through the solid. The heat conduction through the solid material solved by using Eq. 6 is referred to as "conduction coupled with radiative emission" in this work. The extra term $\nabla \cdot q_{\mathrm{rad}}$ in the Eq. 6 is added into source term vector $\mathbf{S}$ of Eq. 1 and is calculated as

$$
\nabla \cdot q_{\mathrm{rad}}=\int_{\lambda} \nabla \cdot q_{\lambda} d \lambda=\int_{\lambda} \kappa_{\lambda}\left(4 \pi I_{b \lambda}-G_{\lambda}\right) d \lambda
$$

At each time step, Eq. 4 is solved for $G_{\lambda}$ by using the temperature distribution from the previous time step. Since Eq. 4 represents spectral properties, the number of equations solved at each cell center is equivalent to the chosen wavelength intervals. The net emission is then calculated using Eq. 7, and Eq. 6 is solved with $\nabla \cdot q_{\text {rad }}$ inserted into it. With the new temperature distribution as the output, Eq. 4 is solved for new $G_{\lambda}$. This process is repeated until the difference between the temperature distributions, for the successive iterations, is below the prescribed tolerance.

The data for spectral radiative flux generally exists over a wide range of grid points (or wavelengths). Therefore, the different methodologies used to solve Eq. 4 can be broadly classified into three models: Full Spectrum Resolved model, Kappa Resolved model, and Band model.

\section{II.B.1. Full Spectrum Resolved model}

The Full Spectrum Resolved model solves Eq. 4 by taking the grid points of spectral data as the number of wavelength intervals. It should be noted that the number of equations solved using this model is directly dependent on the spectral data. Since the number of grid points for the spectrum is very high (usually in millions), it is computationally expensive to use this model. However, the solution obtained through this model is the most accurate version and can be used as a benchmark for the validation of other models.

\section{II.B.2. Kappa Resolved model}

The absorption coefficient plays a significant role in the spectral radiative heat transfer problems due to its substantial variation of values along the spectrum. The material's radiative properties (absorption and extinction coefficients) are derived experimentally. Figure 1 shows the experimentally derived absorption coefficient values for LI-900 material. It can be deduced from Fig. 1 that the value of absorption coefficient between any two experimental points can be derived through interpolation. Therefore, the minimum number of equations considered for solving is equal to the number of absorption coefficient experimental points of the material. Unlike hot gases, the radiative properties of solid materials are only wavelength-dependent. It should be noted that the Kappa Resolved model depends on the material properties compared to the Full Spectrum Resolved model, which relies on spectral data. In the earlier work, ${ }^{4}$ Kappa Resolved model was used for coupled scheme simulations.

\section{II.B.3. Band model}

Band model is an extension to Kappa Resolved model, where the spectral domain is divided into small and unequal width bands. Therefore, integration of Eq. 7 over the entire spectrum can be expressed as a summation over bands:

$$
\nabla \cdot q_{\mathrm{rad}}=\int_{0}^{\infty} \nabla \cdot q_{\lambda} d \lambda=\sum_{i=1}^{N} \int_{\lambda_{1}^{B_{i}}}^{\lambda_{2}^{B_{i}}} \nabla \cdot q_{\lambda} d \lambda=\sum_{i=1}^{N} \nabla \cdot q_{B_{i}}
$$

where $N$ represents the number of bands, $B_{i}$ represents the Band number, $\left[\lambda_{1}^{B_{i}}, \lambda_{2}^{B_{i}}\right]$ denote wavelength interval for Band ' $i$ ', and $\nabla \cdot q_{B_{i}}$ represents the mean divergence of heat flux for band ' $i$ ' which is expressed as

$$
\nabla \cdot q_{B_{i}}=\int_{\lambda_{1}^{B_{i}}}^{\lambda_{2}^{B_{i}}} \kappa_{\lambda}\left(4 \pi I_{b \lambda}-G_{\lambda}\right) d \lambda=\kappa_{B_{i}}\left(4 \pi I_{b B_{i}}-G_{B_{i}}\right)
$$




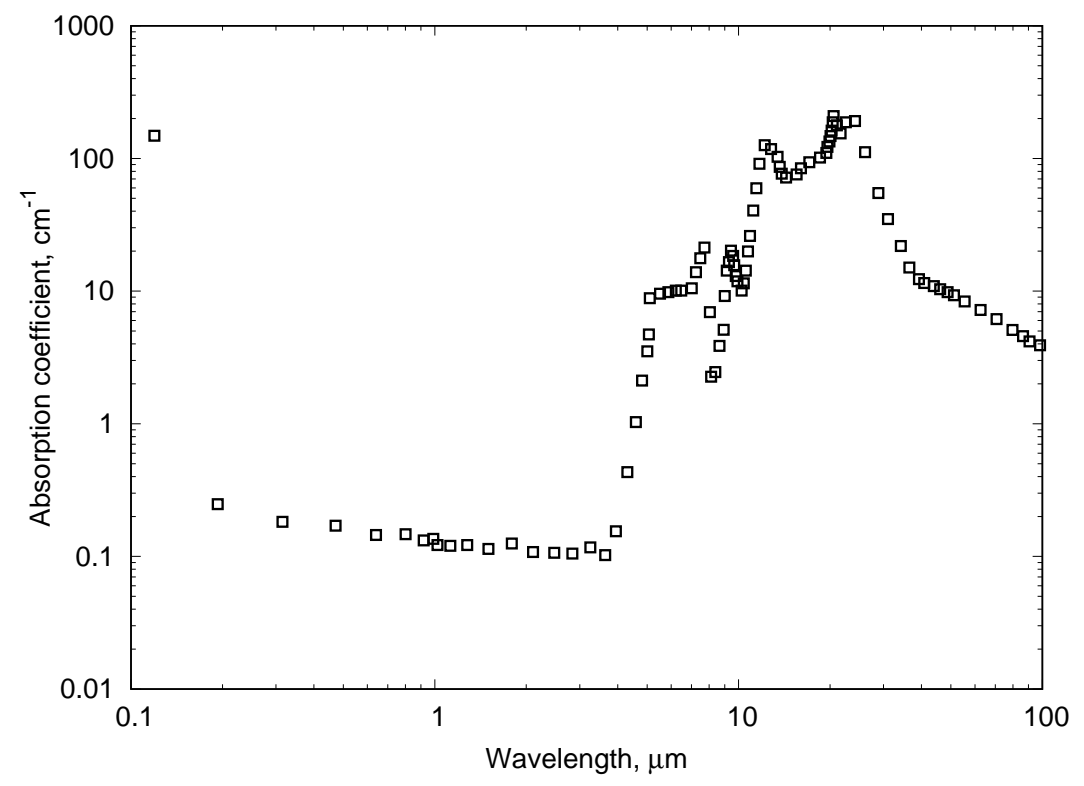

Figure 1. Absorption coefficients of LI-900 material

where $\kappa_{B_{i}}, I_{b B_{i}}$, and $G_{B_{i}}$ denote mean absorption coefficient, mean Planck function, and mean spectral incident flux for Band ' $i$ ', respectively. Similarly, Eq. 3 for each band can be rewritten as

$$
q_{B_{i}}=\int_{\lambda_{1}^{B_{i}}}^{\lambda_{2}^{B_{i}}} q_{\lambda} d \lambda=\int_{\lambda_{1}^{B_{i}}}^{\lambda_{2}^{B_{i}}}-\frac{1}{3 \beta_{\lambda}} \nabla G_{\lambda} d \lambda=-\frac{1}{3 \beta_{B_{i}}} \nabla G_{B_{i}}
$$

where $q_{B_{i}}$ is the mean spectral radiative flux and $\beta_{B_{i}}$ is the mean extinction coefficient for Band ' $i$ ' respectively. The mean Planck function and mean spectral incident flux for Band ' $i$ ' can be expressed as

$$
I_{b B_{i}}=\int_{\lambda_{1}^{B_{i}}}^{\lambda_{2}^{B_{i}}} I_{b \lambda} d \lambda \quad G_{B_{i}}=\int_{\lambda_{1}^{B_{i}}}^{\lambda_{2}^{B_{i}}} G_{\lambda} d \lambda
$$

The average of all the $\kappa_{\lambda}$ and $\beta_{\lambda}$ present in that particular wavelength interval of Band ' $i$ ' yields us the mean absorption and extinction coefficients.

However, to have an efficient band model, it is crucial to select an appropriate band number and its corresponding wavelength intervals. Therefore, the wavelength intervals are manually selected initially, and statistical analysis on absorption coefficient data is performed until an optimum band number is derived. The mean extinction coefficients are then derived based on the wavelength intervals.

In this work, Band model is used to simulate the conduction coupled with radiative emission. The radiative properties ${ }^{21}$ of LI-900 are used for this work. Therefore, a Band model for LI-900 is derived which is presented in Fig. 2. It is a 18-band model. It should be noted that the last band ranges from $98 \mu \mathrm{m}$ to $100 \mu \mathrm{m}$ which does not appear clearly in Fig. 2.

Though the presented Band model corresponds to LI-900 only, similar models can be constructed for other materials using the same methodology.

\section{Results and Discussions}

\section{III.A. Verification}

A verification study is performed to ensure that Band model simulates the conduction coupled with radiative emission accurately. Two cases are considered for the verification: Marschall ${ }^{21}$ case and coupled scheme on artificial spectrum. 


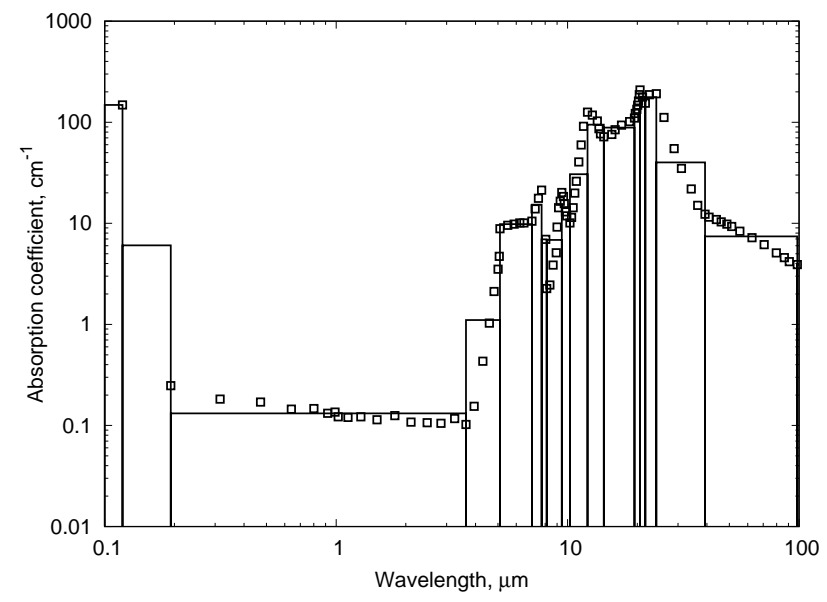

(a) Absorption coefficient

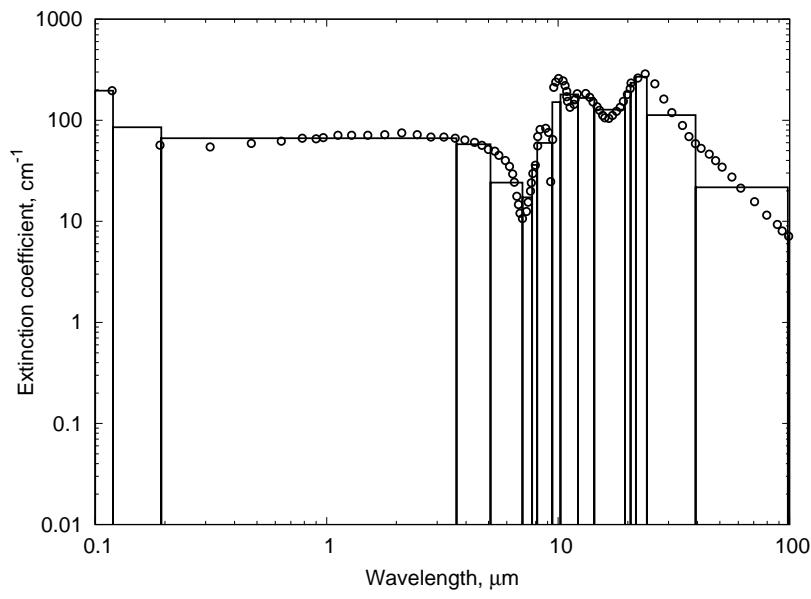

(b) Extinction coefficient

Figure 2. Band Model for LI-900

\section{III.A.1. Marschall case}

In order to check if the Band model simulates effectively, the Radiant heating case by Marschall et al. ${ }^{21}$ is used. A $1.25 \mathrm{~cm}$ of LI-900 sample is heated from $256 \mathrm{~K}$ to $1228 \mathrm{~K}$ at 1 atmosphere for 15 seconds and remains at $1228 \mathrm{~K}$ for the rest of the heating time. Pure conduction and conduction coupled radiative emission using both Kappa Resolved model and Band model are simulated and compared. The comparative results are presented in Fig. 3. The temperature profiles for coupling scheme using Band model appears to agree well as seen in Fig. 3.

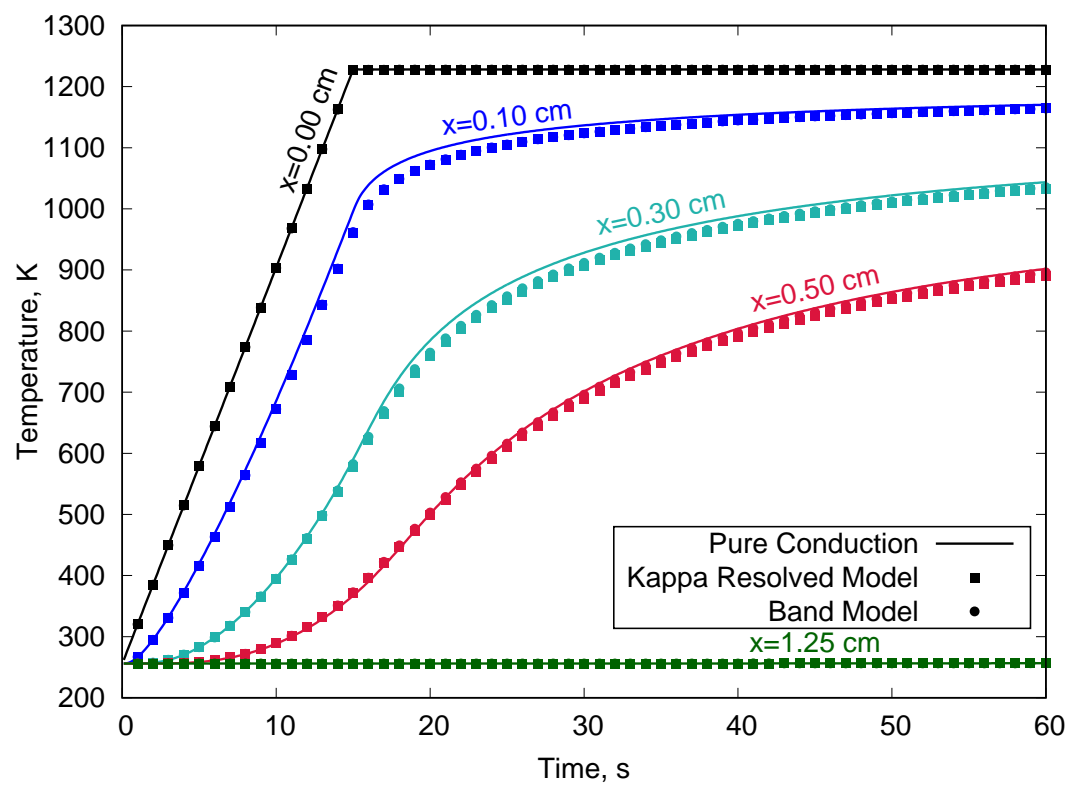

Figure 3. Comparison between pure conduction and conduction coupled with radiative emission for radiant heating case

\section{III.A.2. Conduction coupled radiative emission on manufactured spectrum}

In order to check if the Band model simulates spectral radiative heat problems accurately, an artificial spectrum is constructed. Figure 4(a) represents the manufactured spectrum with 3169 grid points. The 
thermal response of LI-900 sample is simulated using all the three models (Full Spectrum Resolved model, Kappa Resolved model, and Band model). Since Full Spectrum Resolved model acts as a benchmark solution, the closeness of solutions simulated by other models determine their accuracy. Figure 4(b) presents the temperature profiles for all three models. It can be seen that both the Band model and Kappa Resolved model accurately predict the temperature distributions within the material.

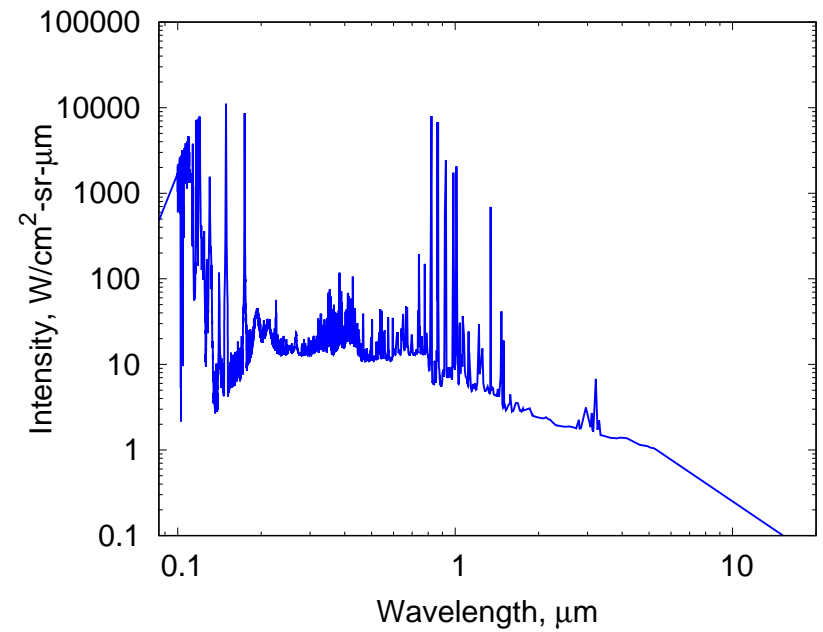

(a) Manufactured spectrum

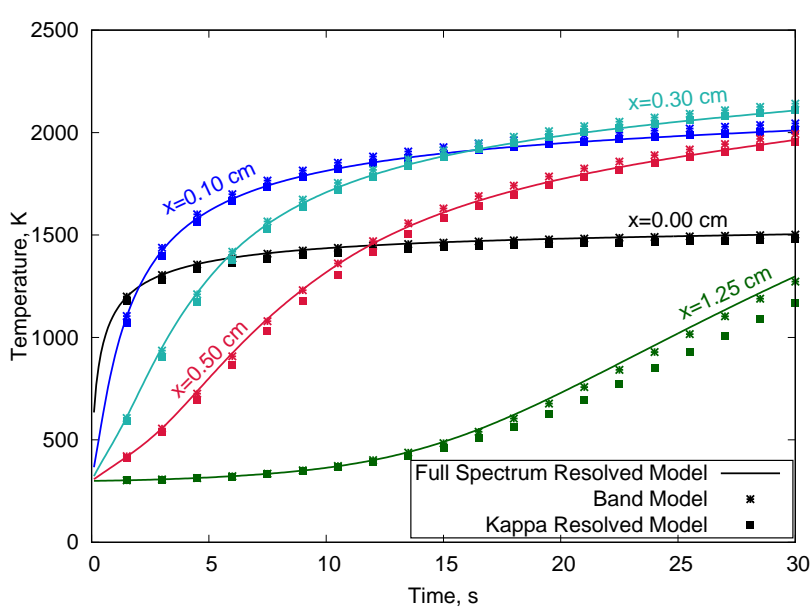

(b) Temperature profiles at various locations withing the sample simulated by different models

Figure 4. Comparison between different models for simulating the thermal response of the material when exposed to a manufactured spectral radiative heat flux

Based on the verification results, it can be inferred that both Kappa Resolved model and Band model simulate accurate thermal response of the material. However, since the number of spectral equations used in the Band model is relatively minor, it proves that it is computationally efficient. Hence, the Band model is considered in this work to simulate the material's response when the spectral radiative heat flux is incident on it.

\section{III.B. Effect of spectral radiative flux}

The response of the material, when exposed to spectral radiative heat flux, is simulated to evaluate its effect. The material used for simulations is a combination of two materials. The thermophysical properties correspond to TACOT, ${ }^{22}$ a fictitious material based on PICA, whereas radiative properties of LI- $900^{21}$ are taken. The simulation is run for a $2 \mathrm{~cm}$ sample.

The spectral data ${ }^{23}$ considered for the simulation is shown in Fig. 5 which consists of around 2.6 million grid points. It can be seen that spectral intensity for different grid points is shown in Fig. 5(a), whereas the cumulative intensity (or Radiant power) over the spectral domain is represented in Fig. 5(b). It should be noted that for a pure conduction simulation, the total radiative heat flux, which is the last point of the data associated with Fig. 5(b), is given as a boundary condition. On the other hand, the cumulative spectral intensities over the respective wavelength interval of the band are used as a boundary condition for conduction coupled radiative emission.

The results from the simulations are showcased in Fig. 6. The temperature and density profiles at various locations within the sample, as a function of heating time, are shown in Fig. 6(a) and (b). It can be seen that the temperature simulated by pure conduction at the heating surface is higher than the one predicted by the Band model. However, the internal temperatures are higher when we consider spectral radiation. The spectral form of energy is not totally absorbed at the heating surface but is traveled through the sample and deposited inside. It should also be noted that the spectral intensities for the wavelength that have high magnitude, as shown in Fig. 5, corresponds to comparatively low absorption coefficients of LI-900 as seen in Fig. 1. The energy corresponding to the high absorption coefficient wavelength gets totally absorbed at the heating surface. Conversely, the energy corresponding to a low absorption coefficient penetrates through the material until getting totally absorbed. The results also indicate the significant difference in 


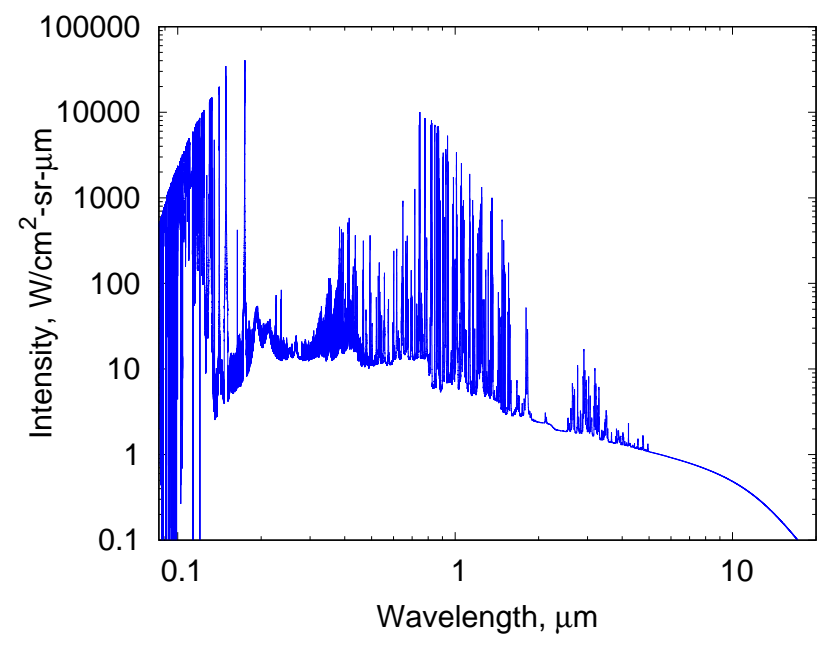

(a) Spectral Intensity

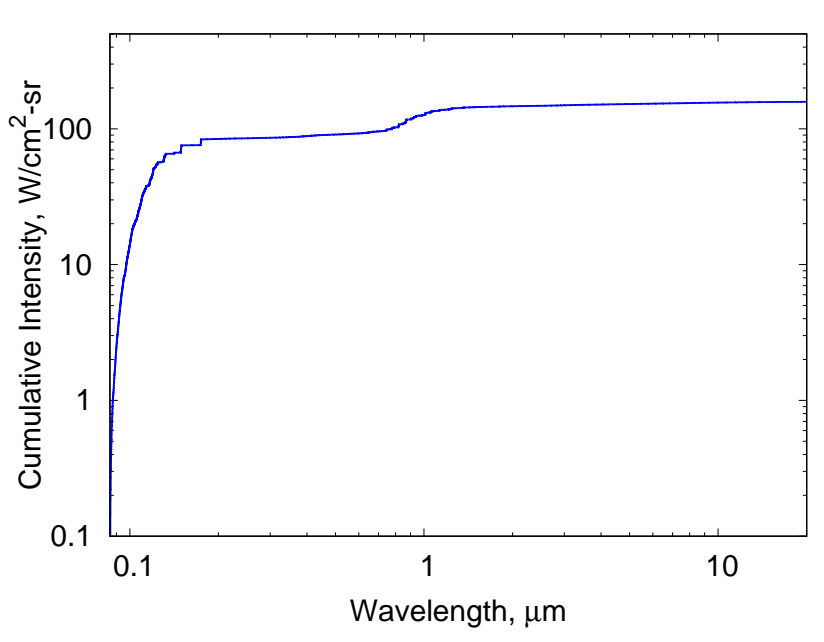

(b) Radiant Power

Figure 5. Spectral intensity and Radiant power of the spectrum

material decomposition for the two methods, as shown in Fig. 6(b). It can be seen that the decomposition of some locations within the material takes place much earlier when considering spectral flux. Since solid decomposition is a function of temperature, the behavior at various locations seems to follow the same trend as temperature profiles, as shown in Fig. 6(a).

The temperature and density distributions along the sample length at the end of 15 seconds of heating time are presented in Fig. 6(c) and (d). The internal temperatures are high, and the material is decomposed more when the effect of spectral radiative heat flux is considered. The extent to which the energy is deposited can also be understood from the magnitude of the radiation source term along the sample length, as shown in Fig. 7. It can be seen that by the end of the heating time, the energy is deposited throughout the sample.

The material's response to spectral radiative flux compared to total radiative flux is quite different. It should be noted that this study is performed by considering only the radiative form of heat flux and on a fictitious material. However, the behavior observed from these simulations, which matches with White ${ }^{3}$ experimental observations, can be treated as a general trend. The spectral radiative flux impacts the material response by depositing energy within the material, especially around the wavelength range that corresponds to the low absorption coefficient of the material.

\section{Conclusion}

A coupling scheme between the material response module of KATS and $\mathrm{P}_{1}$ approximation of RTE is used to study the effect of spectral radiative heat flux. A Band model was constructed for LI-900 and was verified by comparing the simulated results with both pure conduction results and results computed by other models of RTE. The comparisons agree well, inferring that the Band model is computationally efficient in handling the spectral data.

In order to evaluate the impact, simulations for the response of the material exposed to spectral radiative flux were run. The results of pure conduction and conduction coupled radiative emission using the Band model were compared, indicating significant differences in temperature and density distributions. The results computed using the Band model showed that the energy was deposited inside the material, thereby changing the internal distributions completely. The material would possess higher internal temperatures and earlier internal decomposition when the spectral part of the radiation is taken into account. The behaviors from the study indicate that the spectral radiative flux does impact the material response. 

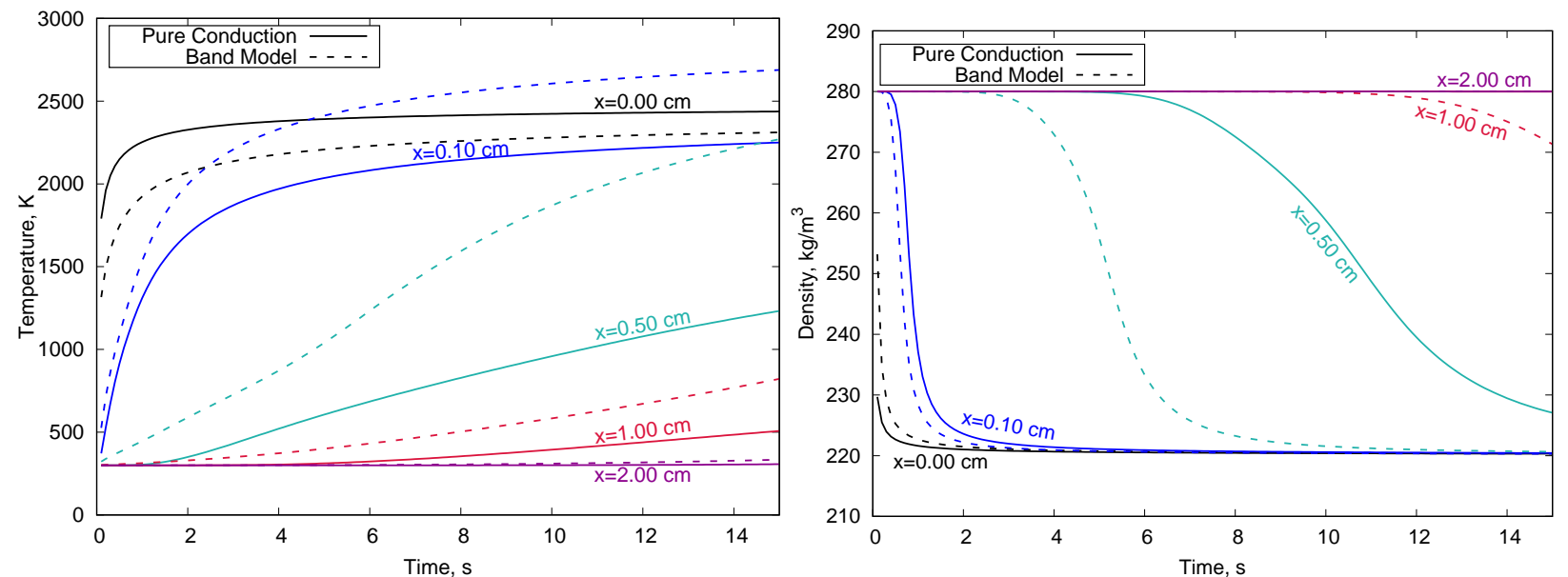

(a) Temperature profiles at various locations within the sample

(b) Density profiles at various locations within the sample as as a function of time a function of time
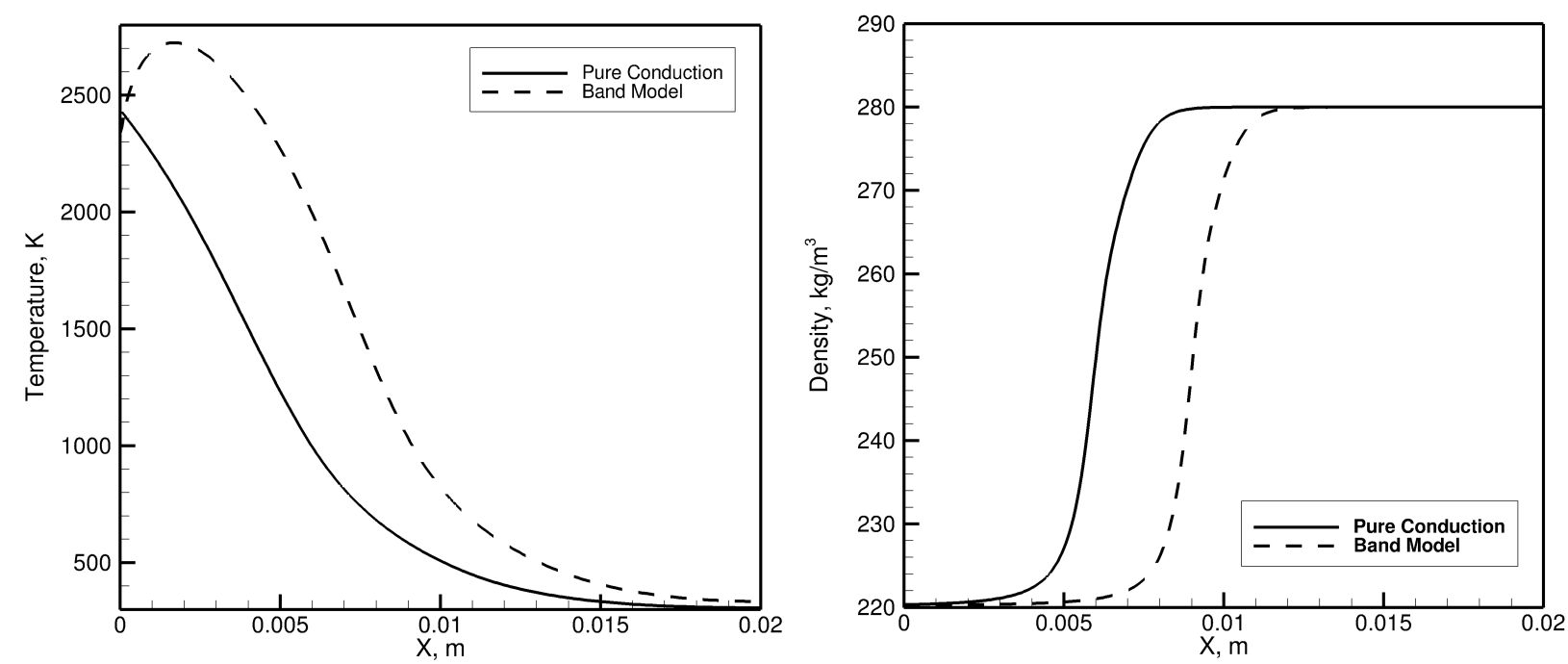

(c) Temperature distribution along the sample at the end of (d) Density distribution along the sample at the end of the heating time heating time

Figure 6. Temperature and solid density profiles as a function of both time and length 


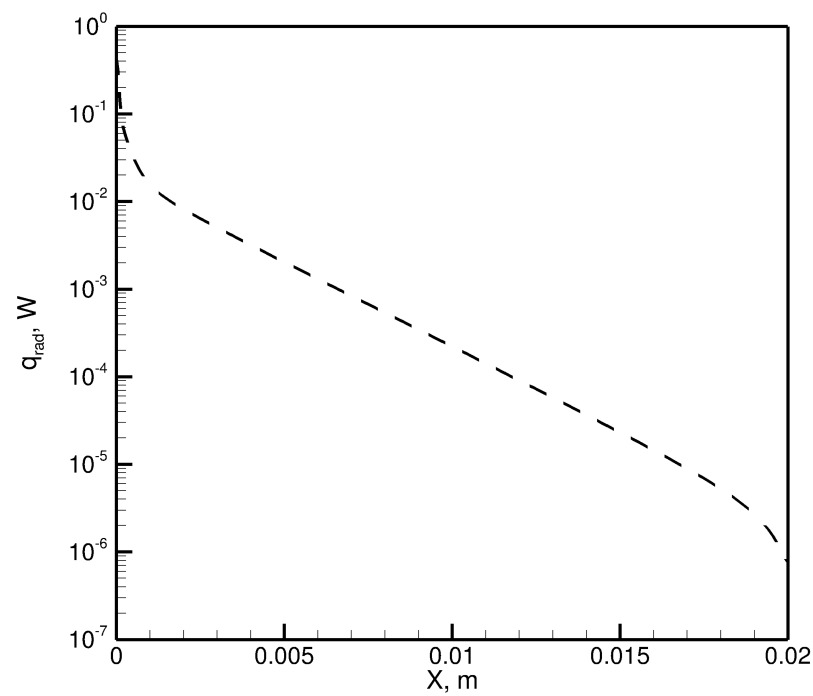

Figure 7. Radiation source term distribution along the sample at the end of heating time

\section{Acknowledgements}

Financial support for this work was provided by the NASA SpaceTech - REDDI - 2018 - ESI grant 80NSSC19K0218. The authors would like to thank Aaron Brandis at AMA, Inc. for assistance and insightful discussions. The first author would also like to thank Savio Poovathingal and Ayan Banerjee at the University of Kentucky for assistance.

\section{References}

${ }^{1}$ Bowman, W. H. and Lawrence, R. M., "Space Resource. Ablative materials for high-temperature thermal protection of space vehicles," Journal of Chemical Education, Vol. 48, No. 10, 10 1971, pp. 690. doi:10.1021/ed048p690

${ }^{2}$ Lee, S.-C. and George R. Cunnington, J., "Theoretical Models for Radiative Transfer in Fibrous Media," Annual Review of Heat Transfer, Vol. 9, 1998, pp. 159-218. doi:10.1615/AnnualRevHeatTransfer.v9.50

${ }^{3}$ White, S., "Effects of Laser Wavelength on Ablator Testing," 38th Annual Conference on Composites, Materials and Structures, Cocoa Beach, Florida, January 2014. doi:2060/20140011553

${ }^{4}$ Davuluri, R. S. C., Fu, R., Tagavi, K. A., and Martin, A., "Fully coupled internal radiative heat transfer for 3D material response of heat shield," AIAA Aviation 2021 Forum, AIAA Paper 2021-3131, Virtual Event, August 2021. doi:10.2514/6.2021-3131

${ }^{5}$ Bose, D., McCorkle, E., Thompson, C., Bogdanoff, D., Prabhu, D., Allen, G., and Grinstead, J., "Analysis and Model Validation of Shock Layer Radiation in Air," 46th AIAA Aerospace Sciences Meeting and Exhibit, AIAA Paper 2008-1246, Reno, Nevada, January 2008. doi:10.2514/6.2008-1246

${ }^{6}$ Weng, H. and Martin, A., "Multidimensional Modeling of Pyrolysis Gas Transport Inside Charring Ablative Materials," Journal of Thermophysics and Heat Transfer, Vol. 28, No. 4, October 2014, pp. 583-597. doi:10.2514/1.T4434

${ }^{7}$ Davuluri, R. S. C., Zhang, H., and Martin, A., "Numerical Study of Spallation Phenomenon in an Arc-Jet Environment," Journal of Thermophysics and Heat Transfer, Vol. 30, No. 1, January 2016, pp. 32-41. doi:10.2514/1.T4586

${ }^{8}$ Davuluri, R. S. C., Zhang, H., and Martin, A., "Effects of spalled particles thermal degradation on a hypersonic flow field environment," 54th AIAA Aerospace Sciences Meeting, AIAA Paper 2016-0248, San Diego, California, January 2016. doi:10.2514/6.2016-0248

${ }^{9} \mathrm{Fu}$, R., Weng, H., Wenk, J. F., and Martin, A., "Thermomechanical Coupling for Charring Ablators," Journal of Thermophysics and Heat Transfer, Vol. 32, No. 2, April 2018, pp. 369-379. doi:10.2514/1.T5194

${ }^{10} \mathrm{Fu}, \mathrm{R}$., Weng, H., Wenk, J. F., and Martin, A., "Thermal expansion of charring ablative materials," Journal of Thermophysics and Heat Transfer, Vol. 34, No. 1, January 2020, pp. 57-65. doi:10.2514/1.T5718 


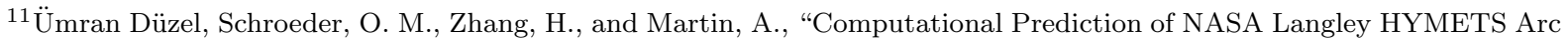
Jet Flow with KATS," 2018 AIAA Aerospace Sciences Meeting, AIAA Paper 2018-1719, American Institute of Aeronautics and Astronautics, Kissimmee, Florida, January 2018. doi:10.2514/6.2018-1719

12 Ümran Düzel and Martin, A., "Modeling High Velocity Flow Through Porous Media," AIAA Scitech 2020 Forum, AIAA Paper 2020-0486, Orlando, Florida, January 2020. doi:10.2514/6.2020-0486

${ }^{13}$ Balay, S., Gropp, W. D., McInnes, L. C., and Smith, B. F., "Efficient Management of Parallelism in Object Oriented Numerical Software Libraries," Modern Software Tools in Scientific Computing, edited by E. Arge, A. M. Bruaset, and H. P. Langtangen, Birkhäuser Press, Boston, MA, 1997. doi:10.1007/978-1-4612-1986-6_8

${ }^{14}$ Balay, S., Brown, J., Buschelman, K., Eijkhout, V., Gropp, W. D., Kaushik, D., Knepley, M. G., McInnes, L. C., Smith, B. F., and Zhang, H., "PETSc Users Manual," Tech. Rep. ANL-95/11 - Revision 3.3, Argonne National Laboratory, 2012.

${ }^{15}$ Balay, S., Abhyankar, S., Adams, M. F., Brown, J., Brune, P., Buschelman, K., Eijkhout, V., Gropp, W. D., Kaushik, D., Knepley, M. G., McInnes, L. C., Rupp, K., Smith, B. F., and Zhang, H., "PETSc Web page," http://www.mcs.anl.gov/petsc, 2014.

${ }^{16}$ Karypis, G. and Kumar, V., "A Fast and High Quality Multilevel Scheme for Partitioning Irregular Graphs," SIAM Journal on Scientific Computing, Vol. 20, No. 1, January 1998, pp. 359-392. doi:10.1137/S1064827595287997

${ }^{17}$ Walker, D. W. and Dongarra, J. J., "MPI: a standard message passing interface," Supercomputer, Vol. 12, No. 1, December 1996, pp. 56-58.

https://www.scopus.com/record/display.uri?eid=2-s2.0-0002789358\&origin=inward\&txGid=b9b115ae3d17f3587282ac510844aea7

${ }^{18}$ Weng, H. and Martin, A., "Numerical Investigation of Thermal Response Using Orthotropic Charring Ablative Material," Journal of Thermophysics and Heat Transfer, Vol. 29, No. 3, July 2015, pp. 429-438. doi:10.2514/1.T4576

${ }^{19}$ Weng, H., Ümran Düzel, Fu, R., and Martin, A., "Geometric Effects on Charring Ablator: Modeling the Full-Scale Stardust Heat Shield," Journal of Spacecraft and Rockets, Vol. 58, No. 2, March 2021, pp. 302-315. doi:10.2514/1.A34828

${ }^{20}$ Modest, M. F., Radiative Heat Transfer, ISBN 978012503163 9, Academic Press, 2nd ed., March 2003.

${ }^{21}$ Marschall, J., Maddren, J., and Parks, J., "Internal Radiation Transport and Effective Thermal Conductivity of Fibrous Ceramic Insulation," 35th AIAA Thermophysics Conference, AIAA Paper 2001-2822, Anaheim, California, June 2001. doi:10.2514/6.2001-2822

${ }^{22}$ Lachaud, J., van Eekelen, T., Bianchi, D., and Martin, A., "TACOT v3.0," Ablation Workshop: Code Comparison, 4, 2014.

doi:10.13023/ablation.test.TACOT

${ }^{23}$ Brandis, A., Private communication. 